\title{
Existence Results for Quasilinear Degenerated Equations Via Strong Convergence of Truncations
}

\author{
Youssef AKDIM, Elhoussine AzRoul, \\ and Abdelmoujib BENKIRANE \\ Département de Mathématiques et Informatique, \\ Faculté des Sciences Dhar-Mahraz, \\ B.P. 1796 Atlas, Fès, Maroc. \\ akdimyoussef@yahoo.fr elazroul@caramail.com \\ abdelmoujib@iam.net.ma
}

Recibido: 29 de Noviembre de 2001

Aceptado: 5 de Febrero de 2004

\begin{abstract}
In this paper we study the existence of solutions for quasilinear degenerated elliptic operators $A(u)+g(x, u, \nabla u)=f$, where $A$ is a Leray-Lions operator from $W_{0}^{1, p}(\Omega, w)$ into its dual, while $g(x, s, \xi)$ is a nonlinear term which has a growth condition with respect to $\xi$ and no growth with respect to $s$, but it satisfies a sign condition on $s$. The right hand side $f$ is assumed to belong either to $W^{-1, p^{\prime}}\left(\Omega, w^{*}\right)$ or to $L^{1}(\Omega)$.
\end{abstract}

Key words: Weighted Sobolev spaces, Hardy inequality, Quasilinear degenerated elliptic operators, Truncations

2000 Mathematics Subject Classification: 35J15, 35J20, 35J70.

\section{Introduction}

In this paper, we shall be concerned with the existence of solutions for quasilinear degenerated elliptic equations of the type

$$
\left\{\begin{array}{l}
A u+g(x, u, \nabla u)=f \quad \text { in } \mathcal{D}^{\prime}(\Omega), \\
u \in W_{0}^{1, p}(\Omega, w), \quad g(x, u, \nabla u) \in L^{1}(\Omega), \quad g(x, u, \nabla u) u \in L^{1}(\Omega),
\end{array}\right.
$$


where

$$
A u=-\operatorname{div}(a(x, u, \nabla u))
$$

is a weighted Leray-Lions operator from the weighted Sobolev space $X=W_{0}^{1, p}(\Omega, w)$ into $X^{*}=W^{-1, p^{\prime}}\left(\Omega, w^{*}\right)$ and where $g$ is a nonlinear lower order term having natural growth $\left(|g(x, s, \xi)| \leq b(|s|)\left(c(x)+\sum_{i=1}^{N} w_{i}\left|\xi_{i}\right|^{p}\right)\right)$ which satisfies the sign condition $g(x, s, \xi) s \geq 0$. The right hand side $f$ is assumed to belong to $X^{*}$ or $L^{1}(\Omega)$. In the last case, we also assume that $g(x, s, \xi)$ has an "exact natural growth" i.e., $|g(x, s, \xi)| \geq$ $\rho \sum_{i=1}^{N} w_{i}\left|\xi_{i}\right|^{p}$. It will turn out that for any solution $u, g(x, u, \nabla u) \in L^{1}(\Omega)$, but for a general $v \in X, g(x, v, \nabla v)$ can be more singular.

Drabek and Nicolosi in [10] proved the existence of bounded solution for the degenerated problem $(\mathcal{P})$ where $g(x, u, \nabla u)=-c_{0}|u|^{p-2} u$, more precisely for the problem,

$$
A u-c_{0}|u|^{p-2} u=f(x, u, \nabla u)
$$

with some more general degeneracy, but under some other assumptions on $f$ and $a(x, s, \xi)$. The existence result for the problem $(\mathcal{P})$ (respectively, unilateral problem) where $f$ lies in the dual space $W^{-1, p^{\prime}}\left(\Omega, w^{*}\right)$ is also studied in [1] (respectively, [2]), namely, the authors obtain the existence results by proving that the positive part $u_{\varepsilon}^{+}$ (resp. $u_{\varepsilon}^{-}$) of $u_{\varepsilon}$ strongly converges to $u^{+}$(resp. $u^{-}$), where $u_{\varepsilon}$ is a solution of the approximate problem.

Our first aim of this paper is to prove (in Theorem 3.7) the same existence result as in [1] by using another approach based on the strong convergence of the truncations $T_{k}\left(u_{\varepsilon}\right)$ in $W_{0}^{1, p}(\Omega, w)$. Moreover, we assume only the weak integrability condition $\sigma^{1-q^{\prime}} \in L_{l o c}^{1}(\Omega)$ (see assumption $\left(H_{1}\right)$ below) instead of the stronger one which is $\sigma^{1-q^{\prime}} \in L^{1}(\Omega)$ as in [1]. For that, we approximate the term $g(x, s, \xi)$ by some functions involving $\chi_{\Omega_{\varepsilon}}$ where $\Omega_{\varepsilon}$ is a sequence of compacts covering the bounded open set $\Omega$ and $\chi_{\Omega_{\varepsilon}}$ is a characteristic function, i.e., $g_{\varepsilon}(x, s, \xi)=\frac{g(x, s, \xi)}{1+\varepsilon|g(x, s, \xi)|} \chi_{\Omega_{\varepsilon}}(x)$.

The second aim of this paper is to prove (in Theorem 3.12) the existence result for the following problem

$$
(\tilde{\mathcal{P}}) \quad\left\{\begin{array}{l}
A u+g(x, u, \nabla u)=f \quad \text { in } \mathcal{D}^{\prime}(\Omega), \\
u \in W_{0}^{1, p}(\Omega, w), \quad g(x, u, \nabla u) \in L^{1}(\Omega),
\end{array}\right.
$$

where $f \in L^{1}(\Omega)$, under some added hypothesis (see (35) below).

Note that in the non weighted case Boccardo, Gallouët and Murat in [6] have proved the existence of at least one solution for the problem $(\mathcal{P})$ and $(\tilde{\mathcal{P}})$. Let us point out that another work in this direction can be found in [4] where the right hand side $f$ is assumed to belong to $W^{-1, p^{\prime}}(\Omega)$ and in [5] with $f \in L^{1}(\Omega)$.

Our results (Theorem 3.7 and Theorem 3.12) generalize those obtained in [4], [5] and [6], in the weighted case.

The present paper is organized as follows: In Section 2, we give some preliminaries. In the first part of Section 3, we prove some technical lemmas concerning some 
convergences in weighted Sobolev spaces and determinate basic assumptions. And in the second part we study the first main result (where $f \in X^{*}$ ). In the third part, we study the case where $f \in L^{1}(\Omega)$. The fifth part is devoted to an example which illustrates our abstract hypotheses. Note that, in the proof of our main general results, many ideas have been adopted from the work of [6].

\section{Preliminaries}

Let $\Omega$ be a bounded open subset of $\mathbb{R}^{N}(N \geq 1)$, let $1<p<\infty$, and let $w=$ $\left\{w_{i}(x), 0 \leq i \leq N\right\}$ be a vector of weight functions; i.e. every component $w_{i}(x)$ is a measurable function which is strictly positive a.e. in $\Omega$. Furthermore, we suppose in all our considerations that

$$
w_{i} \in L_{l o c}^{1}(\Omega)
$$

and

$$
w_{i}^{-\frac{1}{p-1}} \in L_{l o c}^{1}(\Omega)
$$

for any $0 \leq i \leq N$.

We define the weighted space $L^{p}(\Omega, \gamma)$, where $\gamma$ is a weight function on $\Omega$, by

$$
L^{p}(\Omega, \gamma)=\left\{u=u(x), u \gamma^{\frac{1}{p}} \in L^{p}(\Omega)\right\}
$$

with the norm

$$
\|u\|_{p, \gamma}=\left(\int_{\Omega}|u(x)|^{p} \gamma(x) d x\right)^{\frac{1}{p}} .
$$

We denote by $W^{1, p}(\Omega, w)$ the space of all real-valued functions $u \in L^{p}\left(\Omega, w_{0}\right)$ such that the derivatives in the sense of distributions satisfies

$$
\frac{\partial u}{\partial x_{i}} \in L^{p}\left(\Omega, w_{i}\right) \quad \text { for all } i=1, \ldots, N,
$$

which is a Banach space under the norm

$$
\|u\|_{1, p, w}=\left(\int_{\Omega}|u(x)|^{p} w_{0}(x) d x+\sum_{i=1}^{N} \int_{\Omega}\left|\frac{\partial u(x)}{\partial x_{i}}\right|^{p} w_{i}(x) d x\right)^{\frac{1}{p}} .
$$

Since we shall deal with the Dirichlet problem, we shall use the space

$$
X=W_{0}^{1, p}(\Omega, w)
$$

defined as the closure of $C_{0}^{\infty}(\Omega)$ with respect to the norm $(3)$. Note that, $C_{0}^{\infty}(\Omega)$ is dense in $W_{0}^{1, p}(\Omega, w)$ and $\left(X,\|\cdot\|_{1, p, w}\right)$ is a reflexive Banach space.

We recall that the dual space of the weighted Sobolev spaces $W_{0}^{1, p}(\Omega, w)$ is equivalent to $W^{-1, p^{\prime}}\left(\Omega, w^{*}\right)$, where $w^{*}=\left\{w_{i}^{*}=w_{i}^{1-p^{\prime}}, \forall i=0, \ldots, N\right\}$, and $p^{\prime}$ is the conjugate of $p$, i.e. $p^{\prime}=\frac{p}{p-1}$. For more details, we refer the reader to [9].

Now we state the following assumption: 
Assumption $\left(\boldsymbol{H}_{\mathbf{1}}\right)$. The expression

$$
\||u|\|_{X}=\left(\sum_{i=1}^{N} \int_{\Omega}\left|\frac{\partial u(x)}{\partial x_{i}}\right|^{p} w_{i}(x) d x\right)^{\frac{1}{p}}
$$

is a norm defined on $X$ and it is equivalent to the norm (3). There exist a weight function $\sigma$ on $\Omega$ and a parameter $q, \quad 1<q<\infty$, such that

$$
\sigma^{1-q^{\prime}} \in L_{l o c}^{1}(\Omega)
$$

with $q^{\prime}=\frac{q}{q-1}$. The Hardy inequality,

$$
\left(\int_{\Omega}|u(x)|^{q} \sigma d x\right)^{\frac{1}{q}} \leq c\left(\sum_{i=1}^{N} \int_{\Omega}\left|\frac{\partial u(x)}{\partial x_{i}}\right|^{p} w_{i}(x) d x\right)^{\frac{1}{p}}
$$

holds for every $u \in X$ with a constant $c>0$ independent of $u$. Moreover, the imbedding

$$
X \hookrightarrow L^{q}(\Omega, \sigma)
$$

expressed by the inequality (6) is compact.

Note that $\left(X,\||\cdot|\|_{X}\right)$ is a uniformly convex (and thus reflexive) Banach space.

Remark 2.1. Assume that $w_{0}(x) \equiv 1$ and in addition the integrability condition: There exists $\nu \in] \frac{N}{p}, \infty\left[\cap\left[\frac{1}{p-1}, \infty\left[\right.\right.\right.$ such that $w_{i}^{-\nu} \in L^{1}(\Omega)$ for all $i=1, \ldots, N$ (which is stronger than $(2))$. Then

$$
\||u|\|_{X}=\left(\sum_{i=1}^{N} \int_{\Omega}\left|\frac{\partial u(x)}{\partial x_{i}}\right|^{p} w_{i}(x) d x\right)^{\frac{1}{p}}
$$

is a norm defined on $W_{0}^{1, p}(\Omega, w)$ and it is equivalent to (3). Moreover

$$
W_{0}^{1, p}(\Omega, w) \hookrightarrow L^{q}(\Omega)
$$

for all $1 \leq q<p_{1}^{*}$ if $p \nu<N(\nu+1)$ and for all $q \geq 1$ if $p \nu \geq N(\nu+1)$, where $p_{1}=\frac{p \nu}{\nu+1}$ and $p_{1}^{*}=\frac{N p_{1}}{N-p_{1}}=\frac{N p \nu}{N(\nu+1)-p \nu}$ is the Sobolev conjugate of $p_{1}$ (see [9]). Thus the hypotheses $\left(H_{1}\right)$ is satisfied for $\sigma \equiv 1$.

Definition. Let $X$ be a reflexive Banach space. An operator $B$ from $X$ to its dual $X^{*}$ satisfies property $(M)$ if for any sequence $\left(u_{n}\right) \subset X$ satisfying $u_{n} \rightarrow u$ in $X$ weakly, $B\left(u_{n}\right) \rightarrow \chi$ in $X^{*}$ weakly and $\limsup _{n \rightarrow \infty}\left\langle B u_{n}, u_{n}\right\rangle \leq\langle\chi, u\rangle$ then one has $\chi=B(u)$. 


\section{Main results}

Let $A$ be the nonlinear operator from $W_{0}^{1, p}(\Omega, w)$ into its dual $W^{-1, p^{\prime}}\left(\Omega, w^{*}\right)$ defined as

$$
A u=-\operatorname{div}(a(x, u, \nabla u))
$$

where $a: \Omega \times \mathbb{R} \times \mathbb{R}^{N} \longrightarrow \mathbb{R}^{N}$ is a Carathéodory vector-function satisfying the following assumptions:

\section{Assumption $\left(\mathrm{H}_{2}\right)$.}

$$
\begin{gathered}
\left|a_{i}(x, s, \xi)\right| \leq \beta w_{i}^{\frac{1}{p}}(x)\left[k(x)+\sigma^{\frac{1}{p^{\prime}}}|s|^{\frac{q}{p^{\prime}}}+\sum_{j=1}^{N} w_{j}^{\frac{1}{p^{\prime}}}(x)\left|\xi_{j}\right|^{p-1}\right] \quad \text { for } i=1, \ldots, N, \\
{[a(x, s, \xi)-a(x, s, \eta)](\xi-\eta)>0, \text { for all } \xi \neq \eta \in \mathbb{R}^{N},} \\
a(x, s, \xi) \cdot \xi \geq \alpha \sum_{i=1}^{N} w_{i}\left|\xi_{i}\right|^{p}
\end{gathered}
$$

where $k(x)$ is a positive function in $L^{p^{\prime}}(\Omega)$ and $\alpha, \beta$ are positive constants.

Let $g(x, s, \xi)$ be a Carathéodory function satisfying the following assumptions:

Assumption $\left(H_{3}\right)$.

$$
\begin{gathered}
g(x, s, \xi) s \geq 0 \\
|g(x, s, \xi)| \leq b(|s|)\left(\sum_{i=1}^{N} w_{i}\left|\xi_{i}\right|^{p}+c(x)\right),
\end{gathered}
$$

where $b: \mathbb{R}^{+} \longrightarrow \mathbb{R}^{+}$is a continuous increasing function and $c(x)$ a positive function which is in $L^{1}(\Omega)$.

\subsection{Some technical lemmas}

Let us give and prove the following lemmas which are needed below. Note that lemmas 3.1, 3.2, 3.4 and 3.6 are proved in [1]. Nevertheless, for the sake of completeness, we provide their proofs.

Lemma 3.1. Let $g \in L^{r}(\Omega, \gamma)$ and let $g_{n} \in L^{r}(\Omega, \gamma)$, with $\left\|g_{n}\right\|_{r, \gamma} \leq c, 1<r<\infty$. If $g_{n}(x) \longrightarrow g(x)$ a.e. in $\Omega$, then $g_{n} \rightarrow g$ in $L^{r}(\Omega, \gamma)$, where $\rightarrow$ denotes weak convergence and $\gamma$ is a weight function on $\Omega$.

Proof. Since $g_{n} \gamma^{\frac{1}{r}}$ is bounded in $L^{r}(\Omega)$ and $g_{n}(x) \gamma^{\frac{1}{r}}(x) \longrightarrow g(x) \gamma^{\frac{1}{r}}(x)$, a.e. in $\Omega$, then by the Lemma 3.2 [13], we have

$$
g_{n} \gamma^{\frac{1}{r}} \rightarrow g \gamma^{\frac{1}{r}} \quad \text { in } L^{r}(\Omega)
$$


Moreover, for all $\varphi \in L^{r^{\prime}}\left(\Omega, \gamma^{1-r^{\prime}}\right)$, we have $\varphi \gamma^{-\frac{1}{r}} \in L^{r^{\prime}}(\Omega)$. Then

$$
\int_{\Omega} g_{n} \varphi d x \longrightarrow \int_{\Omega} g \varphi d x, \quad \text { i.e. } g_{n} \rightarrow g \text { in } L^{r}(\Omega, \gamma) .
$$

Lemma 3.2. Assume that $\left(H_{1}\right)$ holds. Let $F: \mathbb{R} \longrightarrow \mathbb{R}$ be uniformly Lipschitz, with $F(0)=0$. Let $u \in W_{0}^{1, p}(\Omega, w)$. Then $F(u) \in W_{0}^{1, p}(\Omega, w)$. Moreover, if the set $D$ of discontinuity points of $F^{\prime}$ is finite, then

$$
\frac{\partial(F \circ u)}{\partial x_{i}}= \begin{cases}F^{\prime}(u) \frac{\partial u}{\partial x_{i}} & \text { a.e. in }\{x \in \Omega: u(x) \notin D\}, \\ 0 & \text { a.e. in }\{x \in \Omega: u(x) \in D\} .\end{cases}
$$

Remark 3.3. The previous lemma is a generalization of the corresponding in [11] (pp. 151-152) (where $w \equiv 1$ and $F \in C^{1}(\mathbb{R})$ and $F^{\prime} \in L^{\infty}(\mathbb{R})$ ) and of the corresponding in [3] (where $w_{0} \equiv w_{1} \equiv \cdots \equiv w_{N}$ is some weight function and $F \in C^{1}(\mathbb{R})$ and $\left.F^{\prime} \in L^{\infty}(\mathbb{R})\right)$. Also note that the previous lemma implies that functions in $W_{0}^{1, p}(\Omega, w)$ can be truncated.

Proof of Lemma 3.2. First, note that the proof of the second part of Lemma 3.2 is identical to the corresponding in the non-weighted case (see [11]).

Consider firstly the case $F \in C^{1}(\mathbb{R})$ and $F^{\prime} \in L^{\infty}(\mathbb{R})$. Let $u \in W_{0}^{1, p}(\Omega, w)$. Since $C_{0}^{\infty}(\Omega)$ is dense in $W_{0}^{1, p}(\Omega, w)$, there exists a sequence $u_{n} \in C_{0}^{\infty}(\Omega)$ such that

$$
u_{n} \longrightarrow u \text { in } W_{0}^{1, p}(\Omega, w) .
$$

Passing to a subsequence, we can assume that

$$
u_{n} \longrightarrow u \text { a.e. in } \Omega \text { and } \nabla u_{n} \longrightarrow \nabla u \text { a.e. in } \Omega \text {. }
$$

Then,

$$
F\left(u_{n}\right) \longrightarrow F(u) \text { a.e. in } \Omega .
$$

On the other hand, from the relation $\left|F\left(u_{n}\right)\right|^{p} w_{0} \leq\left\|F^{\prime}\right\|_{\infty}\left|u_{n}\right|^{p} w_{0}$ and

$$
\left|\frac{\partial F\left(u_{n}\right)}{\partial x_{i}}\right|^{p} w_{i}=\left|F^{\prime}\left(u_{n}\right) \frac{\partial u_{n}}{\partial x_{i}}\right|^{p} w_{i} \leq M\left|\frac{\partial u_{n}}{\partial x_{i}}\right|^{p} w_{i}
$$

we deduce that the function $F\left(u_{n}\right)$ remains bounded in $W_{0}^{1, p}(\Omega, w)$. Thus, going to a further subsequence, we obtain

$$
F\left(u_{n}\right) \rightarrow v \text { in } W_{0}^{1, p}(\Omega, w) .
$$

Thanks to (13), (14) and (7) we conclude that

$$
v=F(u) \in W_{0}^{1, p}(\Omega, w) .
$$


We now turn our attention to the general case. Taking convolutions with mollifiers $\rho_{n}$ in $\mathbb{R}$, we have $F_{n}=F * \rho_{n}, F_{n} \in C^{1}(\mathbb{R})$ and $F_{n}^{\prime} \in L^{\infty}(\mathbb{R})$. Then, by the first case we have $F_{n}(u) \in W_{0}^{1, p}(\Omega, w)$. Since $F_{n} \rightarrow F$ uniformly in every compact, we have $F_{n}(u) \rightarrow F(u)$ a.e. in $\Omega$. On the other hand, $\left(F_{n}(u)\right)$ is bounded in $W_{0}^{1, p}(\Omega, w)$, then for a subsequence $F_{n}(u) \rightarrow \bar{v}$ in $W_{0}^{1, p}(\Omega, w)$ and a.e. in $\Omega$ (due to $(7)$ ). Hence,

$$
\bar{v}=F(u) \in W_{0}^{1, p}(\Omega, w) .
$$

The following lemmas follow from the previous lemma.

Lemma 3.4. Assume that $\left(H_{1}\right)$ holds. Let $u \in W_{0}^{1, p}(\Omega, w)$ and let $T_{k}(u), k \in \mathbb{R}^{+}$, the usual truncation. Then $T_{k}(u) \in W_{0}^{1, p}(\Omega, w)$. Moreover, we have

$$
T_{k}(u) \rightarrow u \text { strongly in } W_{0}^{1, p}(\Omega, w) .
$$

Lemma 3.5. Assume that $\left(H_{1}\right)$ holds. Let $\left(u_{n}\right)$ be a sequence of $W_{0}^{1, p}(\Omega, w)$ such that $u_{n} \rightarrow u$ weakly in $W_{0}^{1, p}(\Omega, w)$. Then, $T_{k}\left(u_{n}\right) \rightarrow T_{k}(u)$ weakly in $W_{0}^{1, p}(\Omega, w)$.

Proof. Since $u_{n} \rightarrow u$ in $W_{0}^{1, p}(\Omega, w)$ and by (7) we have for a subsequence $u_{n} \rightarrow u$ strongly in $L^{q}(\Omega, \sigma)$ and a.e. in $\Omega$. On the other hand,

$$
\begin{aligned}
\left\|\left|T_{k}\left(u_{n}\right)\right|\right\|_{X}^{p} & =\sum_{i=1}^{N} \int_{\Omega}\left|\frac{\partial T_{k}\left(u_{n}\right)}{\partial x_{i}}\right|^{p} w_{i}=\sum_{i=1}^{N} \int_{\Omega}\left|T_{k}^{\prime}\left(u_{n}\right) \frac{\partial u_{n}}{\partial x_{i}}\right|^{p} w_{i} \\
& \leq \sum_{i=1}^{N} \int_{\Omega}\left|\frac{\partial u_{n}}{\partial x_{i}}\right|^{p} w_{i}=\left\|\left|u_{n}\right|\right\|_{X}^{p} .
\end{aligned}
$$

Then $\left(T_{k}\left(u_{n}\right)\right)$ is bounded in $W_{0}^{1, p}(\Omega, w)$. Hence, using $(7)$, we have $T_{k}\left(u_{n}\right) \rightarrow T_{k}(u)$ weakly in $W_{0}^{1, p}(\Omega, w)$.

The following lemma generalizes to the weighted case the analogous Lemma 5 in [7]. For that, we use the method of [7] and [13] which gives the strong convergence of $u_{n}$.

Lemma 3.6. Assume that $\left(H_{1}\right)$ and $\left(H_{2}\right)$ are satisfied, and let $\left(u_{n}\right)$ be a sequence in $W_{0}^{1, p}(\Omega, w)$ such that $u_{n} \rightarrow u$ weakly in $W_{0}^{1, p}(\Omega, w)$ and

$$
\int_{\Omega}\left[a\left(x, u_{n}, \nabla u_{n}\right)-a\left(x, u_{n}, \nabla u\right)\right] \nabla\left(u_{n}-u\right) d x \rightarrow 0 .
$$

Then, $u_{n} \rightarrow u$ in $W_{0}^{1, p}(\Omega, w)$.

Proof. Let $D_{n}=\left[a\left(x, u_{n}, \nabla u_{n}\right)-a\left(x, u_{n}, \nabla u\right)\right] \nabla\left(u_{n}-u\right)$. Then, by (9), $D_{n}$ is a positive function and by (15), $D_{n} \rightarrow 0$ in $L^{1}(\Omega)$.

Extracting a subsequence, still denoted by $u_{n}$, and using $(7)$ we can write

$$
u_{n} \rightarrow u \text { a.e. in } \Omega, \quad D_{n} \rightarrow 0 \text { a.e. in } \Omega \text {. }
$$


Then, there exists a subset $B$ of $\Omega$, of zero measure, such that for $x \in \Omega \backslash B$, $|u(x)|<\infty,|\nabla u(x)|<\infty,|k(x)|<\infty, w_{i}(x)>0$ and $u_{n}(x) \rightarrow u(x), D_{n}(x) \rightarrow 0$.

We set $\xi_{n}=\nabla u_{n}(x)$ and $\xi=\nabla u(x)$. Then

$$
\begin{aligned}
D_{n}(x)= & {\left[a\left(x, u_{n}, \xi_{n}\right)-a\left(x, u_{n}, \xi\right)\right]\left(\xi_{n}-\xi\right) } \\
\geq & \alpha \sum_{i=1}^{N} w_{i}\left|\xi_{n}^{i}\right|^{p}+\alpha \sum_{i=1}^{N} w_{i}\left|\xi^{i}\right|^{p} \\
& -\sum_{i=1}^{N} \beta w_{i}^{\frac{1}{p}}\left[k(x)+\sigma^{\frac{1}{p^{\prime}}}\left|u_{n}\right|^{\frac{q}{p^{\prime}}}+\sum_{j=1}^{N} w_{j}^{\frac{1}{p^{j}}}\left|\xi_{n}^{j}\right|^{p-1}\right]\left|\xi^{i}\right| \\
& -\sum_{i=1}^{N} \beta w_{i}^{\frac{1}{p}}\left[k(x)+\sigma^{\frac{1}{p^{\prime}}}\left|u_{n}\right|^{\frac{q}{p^{\prime}}}+\sum_{j=1}^{N} w_{j}^{\frac{1}{p^{\prime}}}\left|\xi^{j}\right|^{p-1}\right]\left|\xi_{n}^{i}\right|
\end{aligned}
$$

i.e.,

$$
D_{n}(x) \geq \alpha \sum_{i=1}^{N} w_{i}\left|\xi_{n}^{i}\right|^{p}-c_{x}\left[1+\sum_{j=1}^{N} w_{j}^{\frac{1}{p^{\prime}}}\left|\xi_{n}^{j}\right|^{p-1}+\sum_{i=1}^{N} w_{i}^{\frac{1}{p}}\left|\xi_{n}^{i}\right|\right]
$$

where $c_{x}$ is a constant which depends on $x$, but does not depend on n. Since $u_{n}(x) \rightarrow$ $u(x)$, we have $\left|u_{n}(x)\right| \leq M_{x}$, where $M_{x}$ is some positive constant. Then, by a standard argument $\left|\xi_{n}\right|$ is bounded uniformly with respect to $n$. Indeed, (16) becomes

$$
D_{n}(x) \geq \sum_{i=1}^{N}\left|\xi_{n}^{i}\right|^{p}\left(\alpha w_{i}-\frac{c_{x}}{N\left|\xi_{n}^{i}\right|^{p}}-\frac{c_{x} w_{i}^{\frac{1}{p^{\prime}}}}{\left|\xi_{n}^{i}\right|}-\frac{c_{x} w_{i}^{\frac{1}{p}}}{\left|\xi_{n}^{i}\right|^{p-1}}\right) .
$$

If $\left|\xi_{n}\right| \rightarrow \infty$ (for a subsequence), there exists at least one $i_{0}$ such that $\left|\xi_{n}^{i_{0}}\right| \rightarrow \infty$, which implies that $D_{n}(x) \rightarrow \infty$ which gives a contradiction.

Let now $\xi^{*}$ be a cluster point of $\xi_{n}$. We have $\left|\xi^{*}\right|<\infty$ and by the continuity of $a$ with respect to the two last variables we obtain

$$
\left(a\left(x, u(x), \xi^{*}\right)-a(x, u(x), \xi)\right)\left(\xi^{*}-\xi\right)=0 .
$$

In view of (9) we have $\xi^{*}=\xi$. The uniqueness of the cluster point implies

$$
\nabla u_{n}(x) \rightarrow \nabla u(x) \text { a.e. in } \Omega .
$$

Since the sequence $a\left(x, u_{n}, \nabla u_{n}\right)$ is bounded in $\prod_{i=1}^{N} L^{p^{\prime}}\left(\Omega, w_{i}^{*}\right)$ and $a\left(x, u_{n}, \nabla u_{n}\right) \rightarrow$ $a(x, u, \nabla u)$ a.e. in $\Omega$, Lemma 3.1 implies

$$
a\left(x, u_{n}, \nabla u_{n}\right) \rightarrow a(x, u, \nabla u) \text { in } \prod_{i=1}^{N} L^{p^{\prime}}\left(\Omega, w_{i}^{*}\right) \text { and a.e. in } \Omega .
$$


We set $\bar{y}_{n}=a\left(x, u_{n}, \nabla u_{n}\right) \nabla u_{n}$ and $\bar{y}=a(x, u, \nabla u) \nabla u$. As in the proof of Lemma 5 in [7] we can write $\bar{y}_{n} \rightarrow \bar{y}$ in $L^{1}(\Omega)$. By (10), we have

$$
\alpha \sum_{i=1}^{N} w_{i}\left|\frac{\partial u_{n}}{\partial x_{i}}\right|^{p} \leq a\left(x, u_{n}, \nabla u_{n}\right) \nabla u_{n}
$$

Let $z_{n}=\sum_{i=1}^{N} w_{i}\left|\frac{\partial u_{n}}{\partial x_{i}}\right|^{p}, z=\sum_{i=1}^{N} w_{i}\left|\frac{\partial u}{\partial x_{i}}\right|^{p}, \quad y_{n}=\frac{\bar{y}_{n}}{\alpha}$ and $y=\frac{\bar{y}}{\alpha}$. Then, by Fatou's lemma we obtain

$$
\int_{\Omega} 2 y d x \leq \liminf _{n \rightarrow \infty} \int_{\Omega} y+y_{n}-\left|z_{n}-z\right| d x
$$

i.e.

$$
0 \leq-\limsup _{n \rightarrow \infty} \int_{\Omega}\left|z_{n}-z\right| d x
$$

hence,

$$
0 \leq \liminf _{n \rightarrow \infty} \int_{\Omega}\left|z_{n}-z\right| d x \leq \limsup _{n \rightarrow \infty} \int_{\Omega}\left|z_{n}-z\right| d x \leq 0 .
$$

This implies

$$
\nabla u_{n} \rightarrow \nabla u \text { in } \prod_{i=1}^{N} L^{p}\left(\Omega, w_{i}\right)
$$

which with (4) completes the present proof.

\subsection{Case where $f \in W^{-1, p^{\prime}}\left(\Omega, w^{*}\right)$}

In this subsection we assume that

$$
f \in W^{-1, p^{\prime}}\left(\Omega, w^{*}\right) .
$$

Consider the nonlinear problem with Dirichlet boundary conditions,

$$
\left\{\begin{array}{l}
A u+g(x, u, \nabla u)=f \text { in } \mathcal{D}^{\prime}(\Omega) \\
u \in W_{0}^{1, p}(\Omega, w), \quad g(x, u, \nabla u) \in L^{1}(\Omega), \quad u g(x, u, \nabla u) \in L^{1}(\Omega) .
\end{array}\right.
$$

Our main result is then the following:

Theorem 3.7. Under the assumptions $\left(H_{1}\right)-\left(H_{3}\right)$ and $(17)$, there exists a solution of $(\mathcal{P})$.

Remarks 3.8. (i) The previous result is also proved in [1] by using another approach based on the strong convergence both of positive and negative parts of the solution $u_{\varepsilon}$ of the approximate problem (see also [4] in non weighted case).

(ii) Theorem 3.7, generalizes to weighted case the analogous statement in [6]. 
(iii) Note that in [1] the authors have assumed that $\sigma^{1-q^{\prime}} \in L^{1}(\Omega)$ which is stronger than (5).

Proof of Theorem 3.7. STEP (1) (The approximate problem and priori estimates) Let $\Omega_{\varepsilon}$ be a sequence of compact subsets of $\Omega$ such that $\Omega_{\varepsilon}$ is increasing to $\Omega$ as $\varepsilon \rightarrow 0$.

We consider the sequence of approximate equations,

$$
\left(\mathcal{P}_{\varepsilon}\right) \quad\left\{\begin{array}{l}
A\left(u_{\varepsilon}\right)+g_{\varepsilon}\left(x, u_{\varepsilon}, \nabla u_{\varepsilon}\right)=f \\
u_{\varepsilon} \in W_{0}^{1, p}(\Omega, w)
\end{array}\right.
$$

where

$$
g_{\varepsilon}(x, s, \xi)=\frac{g(x, s, \xi)}{1+\varepsilon|g(x, s, \xi)|} \chi_{\Omega_{\varepsilon}}(x) .
$$

and where $\chi_{\Omega_{\varepsilon}}$ is the characteristic function of $\Omega_{\varepsilon}$.

Note that $g_{\varepsilon}(x, s, \xi)$ satisfies the following condition

$$
g_{\varepsilon}(x, s, \xi) s \geq 0, \quad\left|g_{\varepsilon}(x, s, \xi)\right| \leq|g(x, s, \xi)| \quad \text { and } \quad\left|g_{\varepsilon}(x, s, \xi)\right| \leq \frac{1}{\varepsilon} .
$$

We define the operator $G_{\varepsilon}: X \longrightarrow X^{*}$ by

$$
\left\langle G_{\varepsilon} u, v\right\rangle=\int_{\Omega} g_{\varepsilon}(x, u, \nabla u) v d x .
$$

Thanks to Hölder's inequality, we have for all $u \in X$ and $v \in X$

$$
\begin{aligned}
\left|\int_{\Omega} g_{\varepsilon}(x, u, \nabla u) v d x\right| & \leq\left(\int_{\Omega}\left|g_{\varepsilon}(x, u, \nabla u)\right|^{q^{\prime}} \sigma^{-\frac{q^{\prime}}{q}} d x\right)^{\frac{1}{q^{\prime}}}\left(\int_{\Omega}|v|^{q} \sigma d x\right)^{\frac{1}{q}} \\
& \leq \frac{1}{\varepsilon}\left(\int_{\Omega_{\varepsilon}} \sigma^{1-q^{\prime}} d x\right)^{\frac{1}{q^{\prime}}}\|v\|_{q, \sigma} \\
& \leq c_{\varepsilon}\||v|\|_{X} .
\end{aligned}
$$

For the above inequality we have used (5) and (7).

Lemma 3.9. The operator $A+G_{\varepsilon}: X \longrightarrow X^{*}$ is bounded, coercive, hemicontinuous and satisfies property $(M)$.

This lemma will be proved below.

In view of Lemma 3.9, Problem $\left(\mathcal{P}_{\varepsilon}\right)$ has a solution by a classical result (cf. Theorem 2.1 and Remark 2.1 in Chapter 2 of [12]). Since $g_{\varepsilon}$ verifies the sign condition, using (10) we obtain

$$
\alpha \sum_{i=1}^{N} \int_{\Omega} w_{i}\left|\frac{\partial u_{\varepsilon}}{\partial x_{i}}\right|^{p} \leq\left\langle f, u_{\varepsilon}\right\rangle,
$$


i.e. $\alpha\left\|\left|u_{\varepsilon}\right|\right\|^{p} \leq\|f\|_{X^{*}}\left\|\left|u_{\varepsilon}\right|\right\|$, then

$$
\left\|\left|u_{\varepsilon}\right|\right\| \leq \beta_{1}
$$

where $\beta_{1}$ is some positive constant.

SteP (2) (Strong convergence of $T_{k}\left(u_{\varepsilon}\right)$ ) Note that many ideas in this step and step (3) have been adapted from the one used in [6].

Thanks to (19) and (7), we can extract a subsequence, still denoted by $u_{\varepsilon}$, such that

$$
u_{\varepsilon} \rightarrow u \text { weakly in } W_{0}^{1, p}(\Omega, w) \text { and } u_{\varepsilon} \longrightarrow u \text { a.e. in } \Omega \text {. }
$$

Let $k>0$, by Lemma 3.5 we have

$$
T_{k}\left(u_{\varepsilon}\right) \rightarrow T_{k}(u) \text { weakly in } W_{0}^{1, p}(\Omega, w) \text { as } \varepsilon \rightarrow 0 .
$$

Our objective is to prove that

$$
T_{k}\left(u_{\varepsilon}\right) \longrightarrow T_{k}(u) \text { strongly in } W_{0}^{1, p}(\Omega, w) \text { as } \varepsilon \rightarrow 0 .
$$

Fix k, and make the notation $z_{\varepsilon}=T_{k}\left(u_{\varepsilon}\right)-T_{k}(u)$. We use as a test function in $\left(\mathcal{P}_{\varepsilon}\right)$ $v_{\varepsilon}=\varphi_{\lambda}\left(z_{\varepsilon}\right)$ where $\varphi_{\lambda}(s)=s e^{\lambda s^{2}}$. Since $v_{\varepsilon}$ is bounded in $X$ and converges to zero a.e. in $\Omega$ and using (7), we have $v_{\varepsilon} \rightarrow 0$ in $X$ as $\varepsilon \rightarrow 0$, then

$$
\left\langle f, v_{\varepsilon}\right\rangle \longrightarrow 0 \text {. }
$$

This implies that

$$
\eta_{1}(\varepsilon)=\left\langle A u_{\varepsilon}, v_{\varepsilon}\right\rangle+\left\langle G_{\varepsilon} u_{\varepsilon}, v_{\varepsilon}\right\rangle=\left\langle f, v_{\varepsilon}\right\rangle \longrightarrow 0 \text { as } \varepsilon \rightarrow 0 .
$$

Since $g_{\varepsilon}\left(x, u_{\varepsilon}, \nabla u_{\varepsilon}\right) v_{\varepsilon} \geq 0$ in the subset $\left\{x \in \Omega,\left|u_{\varepsilon}(x)\right| \geq k\right\}$, statement (23) yields

$$
\left\langle A u_{\varepsilon}, v_{\varepsilon}\right\rangle+\int_{\left\{\left|u_{\varepsilon}\right| \leq k\right\}} g_{\varepsilon}\left(x, u_{\varepsilon}, \nabla u_{\varepsilon}\right) v_{\varepsilon} d x \leq \eta_{1}(\varepsilon) .
$$

We study each term in the left hand side of (24). We have

$$
\begin{aligned}
\left\langle A u_{\varepsilon}, v_{\varepsilon}\right\rangle= & \int_{\Omega} a\left(x, u_{\varepsilon}, \nabla u_{\varepsilon}\right) \nabla\left(T_{k}\left(u_{\varepsilon}\right)-T_{k}(u)\right) \varphi_{\lambda}^{\prime}\left(z_{\varepsilon}\right) d x \\
= & \int_{\Omega} a\left(x, T_{k}\left(u_{\varepsilon}\right), \nabla T_{k}\left(u_{\varepsilon}\right)\right) \nabla\left(T_{k}\left(u_{\varepsilon}\right)-T_{k}(u)\right) \varphi_{\lambda}^{\prime}\left(z_{\varepsilon}\right) d x \\
& -\int_{\left\{\left|u_{\varepsilon}\right|>k\right\}} a\left(x, u_{\varepsilon}, \nabla u_{\varepsilon}\right) \nabla T_{k}(u) \varphi_{\lambda}^{\prime}\left(z_{\varepsilon}\right) d x \\
= & \int_{\Omega}\left(a\left(x, T_{k}\left(u_{\varepsilon}\right), \nabla T_{k}\left(u_{\varepsilon}\right)\right)-a\left(x, T_{k}\left(u_{\varepsilon}\right), \nabla T_{k}(u)\right)\right) \nabla\left(T_{k}\left(u_{\varepsilon}\right)\right. \\
& \left.-T_{k}(u)\right) \varphi_{\lambda}^{\prime}\left(z_{\varepsilon}\right) d x+\eta_{2}(\varepsilon),
\end{aligned}
$$


where

$$
\begin{aligned}
\eta_{2}(\varepsilon) & =\int_{\Omega} a\left(x, T_{k}\left(u_{\varepsilon}\right), \nabla T_{k}(u)\right) \nabla\left(T_{k}\left(u_{\varepsilon}\right)-T_{k}(u)\right) \varphi_{\lambda}^{\prime}\left(z_{\varepsilon}\right) d x \\
& -\int_{\left\{\left|u_{\varepsilon}\right|>k\right\}} a\left(x, u_{\varepsilon}, \nabla u_{\varepsilon}\right) \nabla T_{k}(u) \varphi_{\lambda}^{\prime}\left(z_{\varepsilon}\right) d x
\end{aligned}
$$

which converges to 0 as $\varepsilon \longrightarrow 0$.

On the other hand,

$$
\begin{aligned}
\mid \int_{\left\{\left|u_{\varepsilon}\right| \leq k\right\}} & g_{\varepsilon}\left(x, u_{\varepsilon}, \nabla u_{\varepsilon}\right) v_{\varepsilon} d x\left|\leq \int_{\left\{\left|u_{\varepsilon}\right| \leq k\right\}} b(k)\left[c(x)+\sum_{i=1}^{N}\left|\frac{\partial u_{\varepsilon}}{\partial x_{i}}\right|^{p} w_{i}\right]\right| v_{\varepsilon} \mid d x \\
\leq & b(k) \int_{\left\{\left|u_{\varepsilon}\right| \leq k\right\}} c(x)\left|\varphi_{\lambda}\left(z_{\varepsilon}\right)\right| d x \\
& +\frac{b(k)}{\alpha} \int_{\left\{\left|u_{\varepsilon}\right| \leq k\right\}} a\left(x, u_{\varepsilon}, \nabla u_{\varepsilon}\right) \nabla u_{\varepsilon}\left|\varphi_{\lambda}\left(z_{\varepsilon}\right)\right| d x \\
= & \eta_{3}(\varepsilon)+\frac{b(k)}{\alpha} \int_{\Omega} a\left(x, T_{k}\left(u_{\varepsilon}\right), \nabla T_{k}\left(u_{\varepsilon}\right)\right) \nabla T_{k}\left(u_{\varepsilon}\right)\left|\varphi_{\lambda}\left(z_{\varepsilon}\right)\right| d x \\
= & \frac{b(k)}{\alpha} \int_{\Omega}\left(a\left(x, T_{k}\left(u_{\varepsilon}\right), \nabla T_{k}\left(u_{\varepsilon}\right)\right)-a\left(x, T_{k}\left(u_{\varepsilon}\right), \nabla T_{k}(u)\right)\right) \nabla\left(T_{k}\left(u_{\varepsilon}\right)\right. \\
& \left.-T_{k}(u)\right)\left|\varphi_{\lambda}\left(z_{\varepsilon}\right)\right| d x+\eta_{4}(\varepsilon)
\end{aligned}
$$

where

$$
\eta_{3}(\varepsilon)=b(k) \int_{\left\{\left|u_{\varepsilon}\right| \leq k\right\}} c(x)\left|\varphi_{\lambda}\left(z_{\varepsilon}\right)\right| d x \rightarrow 0 \quad \text { as } \varepsilon \rightarrow 0
$$

and

$$
\begin{aligned}
\eta_{4}(\varepsilon)=\eta_{3}(\varepsilon)+ & \frac{b(k)}{\alpha} \int_{\Omega} a\left(x, T_{k}\left(u_{\varepsilon}\right), \nabla T_{k}(u)\right) \nabla\left(T_{k}\left(u_{\varepsilon}\right)-T_{k}(u)\right)\left|\varphi_{\lambda}\left(z_{\varepsilon}\right)\right| d x+ \\
& \frac{b(k)}{\alpha} \int_{\Omega} a\left(x, T_{k}\left(u_{\varepsilon}\right), \nabla T_{k}\left(u_{\varepsilon}\right)\right) \nabla T_{k}(u)\left|\varphi_{\lambda}\left(z_{\varepsilon}\right)\right| d x \rightarrow 0 \quad \text { as } \varepsilon \rightarrow 0 .
\end{aligned}
$$

Note that, when $\lambda \geq\left(\frac{b(k)}{2 \alpha}\right)^{2}$ we have

$$
\varphi_{\lambda}^{\prime}(s)-\frac{b(k)}{\alpha}|\varphi(s)| \geq \frac{1}{2} .
$$

Combining this with (24), (25) and (26) we obtain

$$
\begin{aligned}
& \int_{\Omega}\left(a\left(x, T_{k}\left(u_{\varepsilon}\right), \nabla T_{k}\left(u_{\varepsilon}\right)\right)-a\right.\left.\left(x, T_{k}\left(u_{\varepsilon}\right), \nabla T_{k}(u)\right)\right) \nabla\left(T_{k}\left(u_{\varepsilon}\right)-T_{k}(u)\right) d x \leq \\
& \leq \eta_{5}(\varepsilon)=2\left(\eta_{1}(\varepsilon)-\eta_{2}(\varepsilon)+\eta_{4}(\varepsilon)\right) \rightarrow 0 \quad \text { as } \varepsilon \rightarrow 0 .
\end{aligned}
$$


Finally, Lemma 3.6 implies (21).

SteP (3) (Passing to the limit) In virtue of (21) we have for a subsequence

$$
\nabla u_{\varepsilon} \rightarrow \nabla u \quad \text { a.e. in } \Omega,
$$

which with (20) yields

$$
\begin{cases}a\left(x, u_{\varepsilon}, \nabla u_{\varepsilon}\right) \rightarrow a(x, u, \nabla u) & \text { a.e. in } \Omega \\ g_{\varepsilon}\left(x, u_{\varepsilon}, \nabla u_{\varepsilon}\right) \rightarrow g(x, u, \nabla u) & \text { a.e. in } \Omega \\ g_{\varepsilon}\left(x, u_{\varepsilon}, \nabla u_{\varepsilon}\right) u_{\varepsilon} \rightarrow g(x, u, \nabla u) u & \text { a.e. in } \Omega .\end{cases}
$$

On the other hand, thanks to (8) and (19), we have that $a\left(x, u_{\varepsilon}, \nabla u_{\varepsilon}\right)$ is bounded in $\prod_{i=1}^{N} L^{p^{\prime}}\left(\Omega, w_{i}^{*}\right)$. Then, by Lemma 3.1 , we obtain

$$
a\left(x, u_{\varepsilon}, \nabla u_{\varepsilon}\right) \rightarrow a(x, u, \nabla u) \quad \text { weakly in } \prod_{i=1}^{N} L^{p^{\prime}}\left(\Omega, w_{i}^{*}\right) .
$$

It remains to prove that

$$
g_{\varepsilon}\left(x, u_{\varepsilon}, \nabla u_{\varepsilon}\right) \rightarrow g(x, u, \nabla u) \quad \text { strongly in } L^{1}(\Omega) .
$$

By (27), applying Vitali's theorem it suffices to prove that $g_{\varepsilon}\left(x, u_{\varepsilon}, \nabla u_{\varepsilon}\right)$ is uniformly equi-integrable. Indeed, multiplying $\left(\mathcal{P}_{\varepsilon}\right)$ by $u_{\varepsilon}$ and thanks to $(10),(11)$ and (19), we obtain

$$
0 \leq \int_{\Omega} g_{\varepsilon}\left(x, u_{\varepsilon}, \nabla u_{\varepsilon}\right) u_{\varepsilon} d x \leq \tilde{\beta},
$$

where $\tilde{\beta}$ is some positive constant.

For any measurable subset $E$ of $\Omega$ and any $m>0$, we have

$$
\int_{E}\left|g_{\varepsilon}\left(x, u_{\varepsilon}, \nabla u_{\varepsilon}\right)\right| d x=\int_{E \cap X_{m}^{\varepsilon}}\left|g_{\varepsilon}\left(x, u_{\varepsilon}, \nabla u_{\varepsilon}\right)\right| d x+\int_{E \cap Y_{m}^{\varepsilon}}\left|g_{\varepsilon}\left(x, u_{\varepsilon}, \nabla u_{\varepsilon}\right)\right| d x
$$

where

$$
X_{m}^{\varepsilon}=\left\{x \in \Omega,\left|u_{\varepsilon}(x)\right| \leq m\right\}, \quad Y_{m}^{\varepsilon}=\left\{x \in \Omega,\left|u_{\varepsilon}(x)\right|>m\right\}
$$

From these expressions, (12) and (30), we have

$$
\begin{aligned}
\int_{E}\left|g_{\varepsilon}\left(x, u_{\varepsilon}, \nabla u_{\varepsilon}\right)\right| d x \leq & \int_{E \cap X_{m}^{\varepsilon}}\left|g_{\varepsilon}\left(x, u_{\varepsilon}, \nabla T_{m}\left(u_{\varepsilon}\right)\right)\right| d x \\
& +\frac{1}{m} \int_{\Omega} g_{\varepsilon}\left(x, u_{\varepsilon}, \nabla u_{\varepsilon}\right) u_{\varepsilon} d x \\
\leq & b(m) \int_{E}\left(\sum_{i=1}^{N} w_{i}\left|\frac{\partial T_{m}\left(u_{\varepsilon}\right)}{\partial x_{i}}\right|^{p}+c(x)\right) d x+\tilde{\beta} \frac{1}{m}
\end{aligned}
$$


Since the sequence $\left(\nabla T_{m}\left(u_{\varepsilon}\right)\right)$ converges strongly in $\prod_{i=1}^{N} L^{p}\left(\Omega, w_{i}\right)$, the above inequality implies the equi-integrability of $g_{\varepsilon}\left(x, u_{\varepsilon}, \nabla u_{\varepsilon}\right)$. From (28) and (29), we can pass to the limit in

$$
\left\langle A u_{\varepsilon}, v\right\rangle+\int_{\Omega} g_{\varepsilon}\left(x, u_{\varepsilon}, \nabla u_{\varepsilon}\right) v=\langle f, v\rangle
$$

and we obtain,

$$
\langle A u, v\rangle+\int_{\Omega} g(x, u, \nabla u) v=\langle f, v\rangle \quad \text { for any } v \in W_{0}^{1, p}(\Omega, w) \cap L^{\infty}(\Omega) .
$$

Moreover, since $g_{\varepsilon}\left(x, u_{\varepsilon}, \nabla u_{\varepsilon}\right) u_{\varepsilon} \geq 0$ a.e. in $\Omega$, by (27), (30) and Fatou's lemma we have

$$
g(x, u, \nabla u) u \in L^{1}(\Omega) .
$$

This concludes the proof of Theorem 3.7.

Remark 3.10. Note that the statement of (33) holds true for $v=u$, i.e.,

$$
\langle A u, u\rangle+\int_{\Omega} g(x, u, \nabla u) u=\langle f, u\rangle .
$$

Indeed, putting $v=T_{k}(u)$ in (33) and using Lemma 3.4, we have

$$
\left\langle A u-f, T_{k}(u)\right\rangle \rightarrow\langle A u-f, u\rangle .
$$

On the other hand, using Lebesgue's dominated convergence theorem, since

$$
\left|g(x, u, \nabla u) T_{k}(u)\right| \leq|g(x, u, \nabla u)||u| \in L^{1}(\Omega) \quad(\text { due to }(34))
$$

and

$$
g(x, u, \nabla u) T_{k}(u) \rightarrow g(x, u, \nabla u) u \quad \text { a.e. in } \Omega .
$$

we conclude that

$$
g(x, u, \nabla u) T_{k}(u) \rightarrow g(x, u, \nabla u) u \text { in } L^{1}(\Omega) .
$$

Proof of Lemma 3.9. We set $B_{\varepsilon}=A+G_{\varepsilon}$. Using (8) and Hölder's inequality we can show that $A$ is bounded [8]. Thanks to (18) we have $B_{\varepsilon}$ bounded. The coercivity follows from (10) and (11). To show that $B_{\varepsilon}$ is hemicontinuous, let $t \rightarrow t_{0}$, and prove that

$$
\left\langle B_{\varepsilon}(u+t v), \tilde{w}\right\rangle \rightarrow\left\langle B_{\varepsilon}\left(u+t_{0} v\right), \tilde{w}\right\rangle \text { as } t \rightarrow t_{0} \text { for all } u, v, \tilde{w} \in X .
$$

Since for a.e. $x \in \Omega, a_{i}(x, u+t v, \nabla(u+t v)) \rightarrow a_{i}\left(x, u+t_{0} v, \nabla\left(u+t_{0} v\right)\right)$ as $t \rightarrow t_{0}$, thanks to the growth condition (8), Lemma 3.1 implies

$$
a_{i}(x, u+t v, \nabla(u+t v)) \rightarrow a_{i}\left(x, u+t_{0} v, \nabla\left(u+t_{0} v\right)\right) \text { in } L^{p^{\prime}}\left(\Omega, w_{i}^{1-p^{\prime}}\right) \quad \text { as } t \rightarrow t_{0} .
$$


Finally for all $\tilde{w} \in X$,

$$
\langle A(u+t v), \tilde{w}\rangle \longrightarrow\left\langle A\left(u+t_{0} v\right), \tilde{w}\right\rangle \text { as } t \rightarrow t_{0} .
$$

On the other hand, $g_{\varepsilon}(x, u+t v, \nabla(u+t v)) \rightarrow g_{\varepsilon}\left(x, u+t_{0} v, \nabla\left(u+t_{0} v\right)\right)$ as $t \rightarrow t_{0}$ for a.e. in $\Omega$. Also $\left(g_{\varepsilon}(x, u+t v+\nabla(u+t v))\right)_{t}$ is bounded in $L^{q^{\prime}}\left(\Omega, \sigma^{1-q^{\prime}}\right)$ because

$$
\int_{\Omega}\left|g_{\varepsilon}(x, u+t v, \nabla(u+t v))\right|^{q^{\prime}} \sigma^{1-q^{\prime}} \leq\left(\frac{1}{\varepsilon}\right)^{q^{\prime}} \int_{\Omega_{\varepsilon}} \sigma^{1-q^{\prime}} \leq c_{\varepsilon} .
$$

Then, Lemma 3.1 gives

$$
g_{\varepsilon}(x, u+t v, \nabla(u+t v)) \rightarrow g_{\varepsilon}\left(x, u+t_{0} v, \nabla\left(u+t_{0} v\right)\right) \text { in } L^{q^{\prime}}\left(\Omega, \sigma^{1-q^{\prime}}\right) \text { as } t \rightarrow t_{0} .
$$

Since $\tilde{w} \in L^{q}(\Omega, \sigma)$ for all $\tilde{w} \in X$,

$$
\left\langle G_{\varepsilon}(u+t v), \tilde{w}\right\rangle \longrightarrow\left\langle G_{\varepsilon}\left(u+t_{0} v\right), \tilde{w}\right\rangle \text { as } t \longrightarrow t_{0} .
$$

Next we show that $B_{\varepsilon}$ satisfies property (M); i.e. for a sequence $u_{j}$ in $X$ satisfying

(i) $u_{i} \rightarrow u$ in $X$,

(ii) $B_{\varepsilon} u_{j} \rightarrow \chi$ in $X^{*}$ and

(iii) $\lim \sup _{j \rightarrow \infty}\left\langle B_{\varepsilon} u_{j}, u_{j}-u\right\rangle \leq 0$,

we have $\chi=B_{\varepsilon} u$. Indeed, by Hölder's inequality and (7),

$$
\begin{aligned}
\int_{\Omega} g_{\varepsilon}\left(x, u_{j}, \nabla u_{j}\right)\left(u_{j}-u\right) & \leq\left(\int_{\Omega}\left|g_{\varepsilon}\left(x, u_{j}, \nabla u_{j}\right)\right|^{q^{\prime}} \sigma^{\frac{-q^{\prime}}{q}} d x\right)^{\frac{1}{q^{\prime}}}\left(\int_{\Omega}\left|u_{j}-u\right|^{q} \sigma d x\right)^{\frac{1}{q}} \\
& \leq \frac{1}{\varepsilon}\left(\int_{\Omega_{\varepsilon}} \sigma^{\frac{-q^{\prime}}{q}} d x\right)^{\frac{1}{q^{\prime}}}\left\|u_{j}-u\right\|_{q, \sigma} \rightarrow 0 \quad \text { as } j \rightarrow \infty,
\end{aligned}
$$

i.e., $\left\langle G_{\varepsilon} u_{j}, u_{j}-u\right\rangle \rightarrow 0$ as $j \rightarrow \infty$. Combining the last convergence with (iii), we obtain

$$
\limsup _{j \rightarrow \infty}\left\langle A u_{j}, u_{j}-u\right\rangle \leq 0 .
$$

And by the pseudo-monotonicity of $A$ (see Proposition 1 [8]), we have $A u_{j} \rightarrow A u$ in $X^{*}$ and $\lim _{j \rightarrow \infty}\left\langle A u_{j}, u_{j}-u\right\rangle=0$. On the other hand,

$$
\begin{aligned}
0= & \lim _{j \rightarrow \infty} \int_{\Omega} a\left(x, u_{j}, \nabla u_{j}\right) \nabla\left(u_{j}-u\right) d x \\
= & \lim _{j \rightarrow \infty} \int_{\Omega}\left(a\left(x, u_{j}, \nabla u_{j}\right)-a\left(x, u_{j}, \nabla u\right)\right) \nabla\left(u_{j}-u\right) d x \\
& +\int_{\Omega} a\left(x, u_{j}, \nabla u\right) \nabla\left(u_{j}-u\right) d x .
\end{aligned}
$$


The last integral in the right hand tends to zero since $a\left(x, u_{j}, \nabla u\right) \rightarrow a(x, u, \nabla u)$ in $\prod_{i=1}^{N} L^{p^{\prime}}\left(\Omega, w_{i}^{1-p^{\prime}}\right)$ as $j \rightarrow \infty$. Hence, by Lemma 3.6 we have $\nabla u_{j} \rightarrow \nabla u$ a.e. in $\Omega$. Then

$$
g_{\varepsilon}\left(x, u_{j}, \nabla u_{j}\right) \rightarrow g_{\varepsilon}(x, u, \nabla u) \text { a.e. in } \Omega \quad \text { as } j \rightarrow \infty \text {. }
$$

And since

$$
\left|g_{\varepsilon}\left(x, u_{j}, \nabla u_{j}\right) \sigma^{\frac{1-q^{\prime}}{q^{\prime}}}\right| \leq \frac{1}{\varepsilon} \sigma^{\frac{1-q^{\prime}}{q^{\prime}}} \chi_{\Omega_{\varepsilon}} \in L^{q^{\prime}}(\Omega) \quad(\text { due to }(5)),
$$

by Lebesgue's dominated convergence theorem, we obtain

$$
g_{\varepsilon}\left(x, u_{j}, \nabla u_{j}\right) \rightarrow g_{\varepsilon}(x, u, \nabla u) \text { in } L^{q^{\prime}}\left(\Omega, \sigma^{1-q^{\prime}}\right) \text { as } j \rightarrow \infty,
$$

which with (7) implies

$$
\int_{\Omega} g_{\varepsilon}\left(x, u_{j}, \nabla u_{j}\right) v d x \rightarrow \int_{\Omega} g_{\varepsilon}(x, u, \nabla u) v d x \quad \text { as } j \rightarrow \infty, \text { for all } v \in X,
$$

i.e., $G_{\varepsilon} u_{j} \rightarrow G_{\varepsilon} u$ in $X^{*}$. Finally,

$$
B_{\varepsilon} u_{j}=A u_{j}+G_{\varepsilon} u_{j} \rightarrow A u+G_{\varepsilon} u=B_{\varepsilon} u=\chi .
$$

Remark 3.11. The assumption (5) appears necessary in order to prove the boundedness of $G_{\varepsilon}$ in $W_{0}^{1, p}(\Omega, w)$. Thus, when $g \equiv 0$, we don't need to assume $(5)$.

\subsection{The case where $f \in L^{1}(\Omega)$}

In this subsection we assume that

$$
\left\{\begin{array}{l}
f \in L^{1}(\Omega) . \\
\text { There exists } \rho_{1}>0 \text { and } \rho_{2}>0 \text { such that } \\
\quad \text { for }|s| \geq \rho_{1},|g(x, s, \xi)| \geq \rho_{2} \sum_{i=1}^{N} w_{i}\left|\xi_{i}\right|^{p} .
\end{array}\right.
$$

We replace $\left(H_{1}\right)$ by the following assumption

\section{Assumption $\left(H_{1}^{\prime}\right)$.}

$$
\left(H_{1}\right) \text { with } \sigma \in L^{1}(\Omega) \text {. }
$$

Consider the nonlinear problem with Dirichlet boundary conditions

$$
(\tilde{P}) \quad\left\{\begin{array}{l}
A u+g(x, u, \nabla u)=f \text { in } \mathcal{D}^{\prime}(\Omega) \\
u \in W_{0}^{1, p}(\Omega, w), \quad g(x, u, \nabla u) \in L^{1}(\Omega) .
\end{array}\right.
$$

In this case we have the following existence theorem:

Theorem 3.12. Under the assumptions $\left(H_{1}^{\prime}\right),\left(H_{2}\right),\left(H_{3}\right)$ and $(35)$, there exists at least one solution of $(\tilde{\mathcal{P}})$. 
Remark 3.13. Under the assumption (17), $u g(x, u, \nabla u)$ belongs to $L^{1}(\Omega)$, that is not the case in general when we assume the hypothesis (35) (cf. Remark 3 [5]).

Remark 3.14. Theorem 3.12 generalizes to the weighted case the analogous statement in $[5]$ and $[6]$.

Proof of Theorem 3.12. Let $f_{\varepsilon}$ be a sequence of smooth functions which converges strongly to $f$ in $L^{1}(\Omega)$ and $\left\|f_{\varepsilon}\right\|_{L^{1}(\Omega)} \leq c_{1}$ for some constant $c_{1}$.

Now, consider the following approximate problem

$$
\left(\tilde{\mathcal{P}}_{\varepsilon}\right) \quad\left\{\begin{array}{l}
A\left(u_{\varepsilon}\right)+g_{\varepsilon}\left(x, u_{\varepsilon}, \nabla u_{\varepsilon}\right)=f_{\varepsilon} \\
u_{\varepsilon} \in W_{0}^{1, p}(\Omega, w) .
\end{array}\right.
$$

with $g_{\varepsilon}$ is defined as in the problem $\left(\mathcal{P}_{\varepsilon}\right)$. The existence of the solution $u_{\varepsilon}$ of this problem is verified as in the problem $\left(\mathcal{P}_{\varepsilon}\right)$.

Note that the steps of the proof of Theorem 3.12 are similar to those of Theorem 3.7, assuming that the following assertions are verified:

Assertion 1 (Estimate (19)). There exist a constant $c$ such that

$$
\left\|\left|u_{\varepsilon}\right|\right\|_{X} \leq c
$$

where $u_{\varepsilon}$ is a solution of $\left(\tilde{\mathcal{P}}_{\varepsilon}\right)$.

Assertion 2 (Convergence $(22))$. For $v_{\varepsilon}=\varphi_{\lambda}\left(T_{k}\left(u_{\varepsilon}\right)-T_{k}(u)\right)$ where $\varphi_{\lambda}(s)=s e^{\lambda s^{2}}$, we have

$$
\int_{\Omega} f_{\varepsilon} v_{\varepsilon} \rightarrow 0 \text { as } \varepsilon \rightarrow 0
$$

Assertion 3 (Equi-integrability of $\left.\mathbf{g}_{\varepsilon}\left(\mathbf{x}, \mathbf{u}_{\varepsilon}, \nabla \mathbf{u}_{\varepsilon}\right)\right)$. The sequence $\left(g_{\varepsilon}\left(x, u_{\varepsilon}, \nabla u_{\varepsilon}\right)\right)_{\varepsilon}$ is uniformly equi-integrable in $\Omega$.

By applying the assertions described above we deduce the result as in the case when $f \in X^{*}$.

Proof of the assertion 1. Multiplying $\left(\tilde{\mathcal{P}}_{\varepsilon}\right)$ by $T_{k}\left(u_{\varepsilon}\right) \in X$ and since

$$
\int_{\Omega} g_{\varepsilon}\left(x, u_{\varepsilon}, \nabla u_{\varepsilon}\right) T_{k}\left(u_{\varepsilon}\right) \geq 0
$$

we obtain

$$
\int_{\Omega} a\left(x, u_{\varepsilon}, \nabla T_{k}\left(u_{\varepsilon}\right)\right) \nabla T_{k}\left(u_{\varepsilon}\right) \leq \int_{\Omega} f_{\varepsilon} T_{k}\left(u_{\varepsilon}\right) \leq k c_{1} .
$$

In view of (10), we have

$$
\sum_{i=1}^{N} \int_{\Omega} w_{i}\left|\frac{\partial T_{k}\left(u_{\varepsilon}\right)}{\partial x_{i}}\right|^{p} d x \leq \frac{k}{\alpha} c_{1}
$$


i.e.

$$
\left\|\left|T_{k}\left(u_{\varepsilon}\right)\right|\right\|^{p} \leq c_{2}
$$

On the other hand, we have

$$
k \int_{\left\{\left|u_{\varepsilon}\right|>k\right\}}\left|g_{\varepsilon}\left(x, u_{\varepsilon}, \nabla u_{\varepsilon}\right)\right| \leq \int_{\Omega}\left|f_{\varepsilon}\right|\left|T_{k}\left(u_{\varepsilon}\right)\right| d x \leq k c_{1} .
$$

Then, by (35), (36), (37) and for $k>\rho_{1}$, we obtain

$$
\begin{aligned}
\left\|\left|u_{\varepsilon}\right|\right\|_{X}^{p} & =\sum_{i=1}^{N} \int_{\left\{\left|u_{\varepsilon}>k\right|\right\}} w_{i}\left|\frac{\partial u_{\varepsilon}}{\partial x_{i}}\right|^{p} d x+\sum_{i=1}^{N} \int_{\Omega} w_{i}\left|\frac{\partial T_{k}\left(u_{\varepsilon}\right)}{\partial x_{i}}\right|^{p} d x \\
& \leq \frac{1}{\rho_{2}} \int_{\left\{\left|u_{\varepsilon}\right|>k\right\}}\left|g_{\varepsilon}\left(x, u_{\varepsilon}, \nabla u_{\varepsilon}\right)\right|+c_{3}+c_{2} \leq c_{4},
\end{aligned}
$$

where $c_{i}, i=1,2, \ldots$, are various positive constants. Then,

$$
\left\|\left|u_{\varepsilon}\right|\right\| \leq c
$$

Proof of the assertion 2. Since $v_{\varepsilon}$ converges to zero weakly $*$ in $L^{\infty}(\Omega)$. and $f_{\varepsilon}$ converges strongly to $f$ in $L^{1}(\Omega)$. Then,

$$
\int_{\Omega} f_{\varepsilon} v_{\varepsilon} \rightarrow 0 \quad \text { as } \varepsilon \rightarrow 0 .
$$

Proof of the assertion 3. For any measurable subset $E$ of $\Omega$ and any $m>0$, we have, as in (32),

$$
\begin{array}{r}
\int_{E}\left|g_{\varepsilon}\left(x, u_{\varepsilon}, \nabla u_{\varepsilon}\right)\right| d x=\int_{E \cap X_{m}^{\varepsilon}}\left|g_{\varepsilon}\left(x, u_{\varepsilon}, \nabla u_{\varepsilon}\right)\right| d x+\int_{E \cap Y_{m}^{\varepsilon}}\left|g_{\varepsilon}\left(x, u_{\varepsilon}, \nabla u_{\varepsilon}\right)\right| d x \\
\leq b(m) \int_{E}\left(\sum_{i=1}^{N} \omega_{i}\left|\frac{\partial T_{m}\left(u_{\varepsilon}\right)}{\partial x_{i}}\right|^{p}+c(x)\right) d x+\int_{Y_{m}^{\varepsilon}}\left|g_{\varepsilon}\left(x, u_{\varepsilon}, \nabla u_{\varepsilon}\right)\right| d x
\end{array}
$$

For fixed $m$, the first integral of the right hand side of (38) is small uniformly in $\varepsilon$ when the measure of $E$ is small (due to $\nabla T_{m}\left(u_{\varepsilon}\right)$ converges strongly in $\Pi_{i=1}^{N} L^{p}\left(\Omega, w_{i}\right)$ ).

We now discuss the behaviour of the second integral of the right hand side of (38). We use in $\left(\tilde{\mathcal{P}}_{\varepsilon}\right)$ the test function $\psi_{m}\left(u_{\varepsilon}\right)$, where for $m>1$

$$
\begin{cases}\psi_{m}(s)=0 & \text { if }|s| \leq m-1, \\ \psi_{m}(s)=1 & \text { if } s \geq m, \\ \psi_{m}(s)=-1 & \text { if } s \leq-m, \\ \psi_{m}^{\prime}(s)=1 & \text { if } m-1 \leq|s| \leq m\end{cases}
$$


This yields

$$
\int_{\Omega} a\left(x, u_{\varepsilon}, \nabla u_{\varepsilon}\right) \nabla u_{\varepsilon} \psi_{m}^{\prime}\left(u_{\varepsilon}\right) d x+\int_{\Omega} g_{\varepsilon}\left(x, u_{\varepsilon}, \nabla u_{\varepsilon}\right) \psi_{m}\left(u_{\varepsilon}\right) d x=\int_{\Omega} f_{\varepsilon} \psi_{m}\left(u_{\varepsilon}\right) d x,
$$

and thus

$$
\int_{\left\{\left|u_{\varepsilon}\right|>m\right\}}\left|g_{\varepsilon}\left(x, u_{\varepsilon}, \nabla u_{\varepsilon}\right)\right| d x \leq \int_{\left\{\left|u_{\varepsilon}\right|>m-1\right\}}\left|f_{\varepsilon}\right| d x .
$$

From the condition $\sigma \in L^{1}(\Omega)$, it is easy to verify that $\left|\left\{x \in \Omega,\left|u_{\varepsilon}\right|>m-1\right\}\right| \rightarrow$ 0 uniformly in $\varepsilon$ when $m \rightarrow+\infty$, and since $f_{\varepsilon} \rightarrow f$ strongly in $L^{1}(\Omega)$, we have $\int_{\left\{\left|u_{\varepsilon}\right|>m-1\right\}}\left|f_{\varepsilon}\right| d x$ is small uniformly in $\varepsilon$ when $m \rightarrow \infty$, which implies that the second term of the right hand side of (38) is small uniformly in $\varepsilon$ and in $E$, when $m$ is sufficiently large. This completes the proof of the uniform equi-integrability of $g_{\varepsilon}\left(x, u_{\varepsilon}, \nabla u_{\varepsilon}\right)$.

Remark 3.15. The hypothesis $\sigma \in L^{1}(\Omega)$ in $\left(H_{1}^{\prime}\right)$ appears in order to prove that $\left|\left\{x \in \Omega,\left|u_{\varepsilon}\right|>m-1\right\}\right| \rightarrow 0$ uniformly in $\varepsilon$.

\subsection{Example}

Some ideas of this example come from [8]. Let $\Omega$ be a bounded domain of $\mathbb{R}^{N}(N \geq 1)$, satisfying the cone condition. Let us consider the Carathéodory functions:

$$
\begin{gathered}
a_{i}(x, s, \xi)=w_{i}\left|\xi_{i}\right|^{p-1} \operatorname{sgn}\left(\xi_{i}\right) \text { for } i=1, \ldots, N \\
g(x, s, \xi)=\rho s|s|^{r} \sum_{i=1}^{N} w_{i}\left|\xi_{i}\right|^{p}, \quad \rho>0, \quad r>0
\end{gathered}
$$

where $w_{i}(x)(i=0,1, \ldots, N)$ are given weight functions, strictly positive almost everywhere in $\Omega$. We shall assume that the weight functions satisfy, $w_{i}(x)=w(x)$, $x \in \Omega$, for all $i=0, \ldots, N$. Then, we can consider the Hardy inequality (6) in the form,

$$
\left(\int_{\Omega}|u(x)|^{q} \sigma(x) d x\right)^{\frac{1}{q}} \leq c\left(\int_{\Omega}|\nabla u(x)|^{p} w\right)^{\frac{1}{p}} .
$$

It is easy to show that the $a_{i}(x, s, \xi)$ are Carathéodory functions satisfying the growth condition (8) and the coercivity (10). Also the Carathéodory function $g(x, s, \xi)$ satisfies the conditions (11), (12) and (35) with $|s| \geq \rho_{1}=1$ and $\rho_{2}=\rho>0$. On the other hand, the monotonicity condition is satisfied, in fact,

$$
\begin{aligned}
\sum_{i=1}^{N}\left(a_{i}(x, s, \xi)-a_{i}(x, s, \hat{\xi})\right) & \left(\xi_{i}-\hat{\xi}_{i}\right) \\
& =w(x) \sum_{i=1}^{N}\left(\left|\xi_{i}\right|^{p-1} \operatorname{sgn} \xi_{i}-\left|\hat{\xi}_{i}\right|^{p-1} \operatorname{sgn} \hat{\xi}_{i}\right)\left(\xi_{i}-\hat{\xi}_{i}\right)>0
\end{aligned}
$$


for almost all $x \in \Omega$ and for all $\xi, \hat{\xi} \in \mathbb{R}^{N}$ with $\xi \neq \hat{\xi}$, since $w>0$ a.e. in $\Omega$. In particular, let us use the special weight functions $w$ and $\sigma$ expressed in terms of the distance to the boundary $\partial \Omega$. Denote $d(x)=\operatorname{dist}(x, \partial \Omega)$ and set

$$
w(x)=d^{\lambda}(x), \quad \sigma(x)=d^{\mu}(x) .
$$

In this case, the Hardy inequality reads

$$
\left(\int_{\Omega}|u(x)|^{q} d^{\mu}(x) d x\right)^{\frac{1}{q}} \leq c\left(\int_{\Omega}|\nabla u(x)|^{p} d^{\lambda}(x) d x\right)^{\frac{1}{p}} .
$$

The corresponding imbedding is compact if:

(i) For, $1<p \leq q<\infty$,

$$
\lambda<p-1, \quad \frac{N}{q}-\frac{N}{p}+1 \geq 0, \quad \frac{\mu}{q}-\frac{\lambda}{p}+\frac{N}{q}-\frac{N}{p}+1>0 .
$$

(ii) For, $1 \leq q<p<\infty$,

$$
\lambda<p-1, \quad \frac{\mu}{q}-\frac{\lambda}{p}+\frac{1}{q}-\frac{1}{p}+1>0 .
$$

Remark 3.16. Conditions (39) or (40) are sufficient for the compact imbedding (7) to hold (see for example [8, Example 1], [9, Example 1.5, p. 34], and [14, theorems 19.17 and 19.22]).

Finally, the hypotheses of Theorem 3.7 (resp. Theorem 3.12) are satisfied, therefore the problem $(\mathcal{P})(\operatorname{resp} .(\tilde{\mathcal{P}}))$ has at least one solution.

\section{References}

[1] Y. Akdim, E. Azroul, and A. Benkirane, Existence of solutions for quasilinear degenerate elliptic equations, Electron. J. Differential Equations (2001), 1-19.

[2] Existence of solution for quasilinear degenerated elliptic unilateral problems, Ann. Math. Blaise Pascal 10 (2003), 1-20.

[3] B. O. Turesson, Nonlinear potential theory and weighted Sobolev spaces, Lecture Notes in Mathematics, vol. 1736, Springer-Verlag, Berlin, 2000, ISBN 3-540-67588-4.

[4] A. Bensoussan, L. Boccardo, and F. Murat, On a nonlinear partial differential equation having natural growth terms and unbounded solution, Ann. Inst. H. Poincaré Anal. Non Linéaire $\mathbf{5}$ (1988), 347-364. (English, with French summary)

[5] L. Boccardo and T. Gallouët, Strongly nonlinear elliptic equations having natural growth terms and $L^{1}$ data, Nonlinear Anal. 19 (1992), 573-579.

[6] L. Boccardo, T. Gallouët, and F. Murat, A unified presentation of two existence results for problems with natural growth, Progress in Partial Differential Equations: the Metz Surveys, 2 (1992), Pitman Res. Notes Math. Ser., vol. 296, Longman Sci. Tech., Harlow, 1993, pp. 127-137. 
[7] L. Boccardo, F. Murat, and J.-P. Puel, Existence of bounded solutions for nonlinear elliptic unilateral problems, Ann. Mat. Pura Appl. (4) 152 (1988), 183-196. (English, with French and Italian summaries)

[8] P. Drábek, A. Kufner, and V. Mustonen, Pseudo-monotonicity and degenerated or singular elliptic operators, Bull. Austral. Math. Soc. 58 (1998), 213-221.

[9] P. Drábek, A. Kufner, and F. Nicolosi, Non linear elliptic equations, singular and degenerate cases, University of West Bohemia, 1996.

[10] P. Drábek and F. Nicolosi, Existence of bounded solutions for some degenerated quasilinear elliptic equations, Ann. Mat. Pura Appl. (4) 165 (1993), 217-238.

[11] D. Gilbarg and N. S. Trudinger, Elliptic partial differential equations of second order, SpringerVerlag, Berlin, 1977, ISBN 3-540-08007-4.

[12] J.-L. Lions, Quelques méthodes de résolution des problèmes aux limites non linéaires, Dunod, 1969.

[13] J. Leray and Jacques-Louis Lions, Quelques résulatats de Višik sur les problèmes elliptiques nonlinéaires par les méthodes de Minty-Browder, Bull. Soc. Math. France 93 (1965), 97-107.

[14] B. Opic and A. Kufner, Hardy-type inequalities, Pitman Research Notes in Mathematics Series, vol. 219, Longman Scientific \& Technical, Harlow, 1990, ISBN 0-582-05198-3. 